\title{
Steady state sedimentation of ultrasoft colloids
}

Sunil P. Singh, Gerhard Gompper, and Roland G. Winkler

Citation: The Journal of Chemical Physics 148, 084901 (2018); doi: 10.1063/1.5001886

View online: https://doi.org/10.1063/1.5001886

View Table of Contents: http://aip.scitation.org/toc/jcp/148/8

Published by the American Institute of Physics

\section{Articles you may be interested in}

Perspective: Dissipative particle dynamics

The Journal of Chemical Physics 146, 150901 (2017); 10.1063/1.4979514

Stresses in non-equilibrium fluids: Exact formulation and coarse-grained theory

The Journal of Chemical Physics 148, 084503 (2018); 10.1063/1.5019424

Hydrodynamic relaxations in dissipative particle dynamics

The Journal of Chemical Physics 148, 034503 (2018); 10.1063/1.4986569

Communication: Simple liquids' high-density viscosity

The Journal of Chemical Physics 148, 081101 (2018); 10.1063/1.5022058

Perspective: Surface freezing in water: A nexus of experiments and simulations

The Journal of Chemical Physics 147, 060901 (2017); 10.1063/1.4985879

Perspective: Differential dynamic microscopy extracts multi-scale activity in complex fluids and biological systems

The Journal of Chemical Physics 147, 110901 (2017); 10.1063/1.5001027

\section{AIP $\left.\right|^{\text {The Journal of }}$ AIP $\mid$ Chemical Physics} PERSPECTIVES 


\title{
Steady state sedimentation of ultrasoft colloids
}

\author{
Sunil P. Singh, ${ }^{1, \text { a) }}$ Gerhard Gompper, ${ }^{2, b)}$ and Roland G. Winkler ${ }^{2, c)}$ \\ ${ }^{1}$ Indian Institute of Science Education and Research Bhopal, Bhopal By pass Road Bhauri, Bhopal 462066 , \\ Madhya Pradesh, India \\ ${ }^{2}$ Theoretical Soft Matter and Biophysics, Institute for Advanced Simulation and Institute of Complex Systems, \\ Forschungszentrum Jülich, D-52425 Jülich, Germany
}

(Received 28 August 2017; accepted 15 January 2018; published online 22 February 2018)

\begin{abstract}
The structural and dynamical properties of ultra-soft colloids-star polymers-exposed to a uniform external force field are analyzed by applying the multiparticle collision dynamics technique, a hybrid coarse-grain mesoscale simulation approach, which captures thermal fluctuations and longrange hydrodynamic interactions. In the weak-field limit, the structure of the star polymer is nearly unchanged; however, in an intermediate regime, the radius of gyration decreases, in particular transverse to the sedimentation direction. In the limit of a strong field, the radius of gyration increases with field strength. Correspondingly, the sedimentation coefficient increases with increasing field strength, passes through a maximum, and decreases again at high field strengths. The maximum value depends on the functionality of the star polymer. High field strengths lead to symmetry breaking with trailing, strongly stretched polymer arms and a compact star-polymer body. In the weak-field-linear response regime, the sedimentation coefficient follows the scaling relation of a star polymer in terms of functionality and arm length. Published by AIP Publishing. https://doi.org/10.1063/1.5001886
\end{abstract}

\section{INTRODUCTION}

External fields are able to induce drastic conformational changes of soft materials, such as polymers, colloids, vesicles, etc. In turn, their dynamical and transport properties are modified, an effect which can be exploited in technical applications. ${ }^{1,2}$ The understanding of the relation between the nonequilibrium structure and the transport coefficients is fundamental for the rational design of novel functional materials as well as the understanding of the functional principles of biological systems. The intriguing nonequilibrium properties of soft matter in shear and the Poiseuille flow have been illustrated for linear ${ }^{3-13}$ and star polymers ${ }^{14-18}$ as well as vesicles ${ }^{19-27}$ and blood cells. ${ }^{28-37}$

In nature, large macromolecular or colloidal particles sediment to the bottom of a container due to the gravitational force and the density difference of the particles and the solvent. Technically, gravity-driven motion is exploited in analytical ultra-centrifuge techniques for the characterization and separation of synthetic and biological molecules from mixtures. ${ }^{38,39}$ Sedimentation of colloidal and polymeric systems is enormously important for scientific and engineering applications because soft materials whose size and shape are sensitive to thermal fluctuations and weak external flows exhibit interesting and a priori unexpected physical behavior. An example is the sedimentation coefficient of DNA molecules in a dilute suspension, which decreases with the increasing driving force, ${ }^{40}$ denoted as sedimentation anomaly. It is explained by inhomogeneous hydrodynamic interactions of the polymer coil. ${ }^{41-43}$

\footnotetext{
a)Electronic mail: spsingh@iiserb.ac.in

b)Electronic mail: g.gompper@fz-juelich.de

c)Electronic mail: r.winkler@fz-juelich.de
}

The coil exterior, especially the chain ends, experiences a higher drag, while the monomers in the interior are hydrodynamically shielded. This implies a deformation of the coil and a decreasing sedimentation coefficient. ${ }^{41,44}$ Indeed, the computer simulations of Refs. 41 and 44 reveal intriguing conformational changes of the polymer coil with a strong polymer stretching of the trailing end and the formation of a rather compact polymer core.

In the present work, we investigate the steady-state sedimentation properties of dilute suspensions of ultrasoft colloids-star polymers. These colloids are particularly interesting due to their intrinsic nature to inhibit colloidal and polymeric properties. ${ }^{15,17,18,45} \mathrm{~A}$ star polymer is a special type of branched polymer, comprised of several flexible linear polymers which are attached to a common center. The number of polymer arms controls the properties of the colloid-a small number of arms leads to polymer-like behavior and a large number of arms leads to colloidal behavior. The equilibrium and nonequilibrium properties of star polymers have been addressed in various experimental and simulation studies. ${ }^{14-18,46-53}$

Hydrodynamic interactions are essential for the sedimentation of polymers, as discussed, e.g., in Ref. 41. To adequately account for fluid-mediated interactions, we combine molecular dynamics (MD) simulations of a star polymer with the multiparticle collision (MPC) dynamics approach for the fluid. ${ }^{54-56}$ MPC is a particle-based simulation approach, which provides a solution of the Navier-Stokes equations on appropriate length and time scales. ${ }^{57-59}$ It includes thermal fluctuations and is excellently suited for combination with MD simulations. ${ }^{55,56}$ MPC has been shown to provide valuable insight into a broad spectrum of nonequilibrium properties of systems such as polymers, ${ }^{10,11,60-65}$ colloids, ${ }^{48-50,66,68,69}$ vesicles and cells, ${ }^{20,29,33}$ and active particles. ${ }^{69-76}$ 
We find a strong influence of fluid-mediated interactions on the nonequilibrium sedimentation and conformational properties of star polymers. The sedimentation coefficient and the radius of gyration of the star polymer exhibit a nonmonotonic behavior. At intermediate field strengths, the coefficient increases with increasing field strength, assumes a maximum, and decreases at large field strengths again. Thereby, the increase is more pronounced for star polymers with a larger arm number. The changes in the radius of gyration are strongly linked to those of the sedimentation coefficient, however, with the opposite trend, i.e., the radius of gyration decreases first and increases at large field strengths. Interestingly, the star polymers exhibit a trailing tail at high field strengths, with a few strongly stretched polymer arms.

The outline of this paper is as follows. In Sec. II, the starpolymer model, the coarse-grained description of the explicit solvent, and the interaction of the external field with the polymer are introduced. Section III presents results for the conformational and dynamical properties of the star polymers. All results are summarized and discussed in Sec. IV.

\section{MODELS}

\section{A. Star polymer}

We consider a very dilute suspension of star polymers. A star polymer itself consists of $f$ identical flexible linear polymers, which are linked at a common center by one of their ends. A polymer is modeled in a coarse-grained manner as a linear bead-spring chain of $N_{m}$ beads of mass $M$; hence, the total number of beads are $N_{p}=f N_{m}+1$. The bond potential is given by

$$
\begin{aligned}
V_{B}= & \frac{k_{s}}{2} \sum_{\mu=1}^{f} \sum_{k=1}^{N_{m}}\left(\left|\boldsymbol{R}_{k+1}^{\mu}-\boldsymbol{R}_{k}^{\mu}\right|-l\right)^{2} \\
& +\frac{k_{s}}{2} \sum_{\mu=1}^{f}\left(\left|\boldsymbol{R}_{1}^{\mu}-\boldsymbol{R}_{0}\right|-l_{0}\right)^{2},
\end{aligned}
$$

where $\boldsymbol{R}_{k}^{\mu}$ is the position of monomer $k\left(k \in\left\{1, \ldots, N_{m}\right\}\right)$ of arm $\mu(\mu \in\{1, \ldots, f\}), l$ is the equilibrium bond length, and $k_{s}$ denotes the spring constant. The bond length for the central bead $\boldsymbol{R}_{0}$ is $l_{0}$. Excluded-volume interactions between non-bonded beads are taken into account by the repulsive, truncated, and shifted Lennard-Jones (LJ) potential ${ }^{77}$

$$
\begin{aligned}
V_{L J}= & 4 \epsilon \sum_{\nu, \mu=1}^{f} \sum_{k, j=0}^{N_{m}}\left[\left(\frac{\sigma}{R_{k j}^{\nu \mu}}\right)^{12}-\left(\frac{\sigma}{R_{k j}^{v \mu}}\right)^{6}+\frac{1}{4}\right] \\
& \times \Theta\left(2^{1 / 6} \sigma-\Delta R_{k j}^{v \mu}\right) .
\end{aligned}
$$

Here, $\Theta(x)$ is the Heaviside step function $[\Theta(x)=0$ for $x<0$ and $\Theta(x)=1$ for $x \geq 0]$. The distance between the monomers is $R_{k j}^{\nu \mu}=\left|\boldsymbol{R}_{k}^{v}-\boldsymbol{R}_{j}^{\mu}\right|$, with $\boldsymbol{R}_{0}^{v} \equiv \boldsymbol{R}_{0}(v, \mu \in\{1, \ldots$, $\left.f\}, k, j \in\left\{0, \ldots, N_{m}\right\}\right)$. Self-interactions are excluded, i.e., $k \neq j$ for $v=\mu$.

Every star-polymer bead is exposed to the gravitational field $\hat{\boldsymbol{G}}=-\hat{\boldsymbol{G}} \boldsymbol{e}_{y}$, where $\boldsymbol{e}_{y}$ is the unit vector along the direction of the $y$-axis of the Cartesian reference system. Hence, it experiences the force

$$
\boldsymbol{F}_{G}=M \hat{\mathbf{G}} .
$$

In the sedimentation process, fluid is dragged along by a star polymer, which induces a fluid flow. In an experiment, this fluid is reflected by the confining container walls and induces a backflow. To prevent a net fluid flow in our system with periodic boundary conditions, we modify the equations of motion of the fluid in such a way that the total momentum of the system (fluid plus star polymer) is zero. By this requirement, fluid backflow is introduced. This leads to the additional force on a bead (cf. Appendix)

$$
\boldsymbol{F}_{f}=-\frac{M^{2} N_{p}}{M N_{p}+m N_{s}} \hat{\boldsymbol{G}},
$$

where $m$ is the mass of the fluid particle, $N_{s}$ is the total number of fluid particles, and $M N_{p}$ is the total mass of a star polymer.

\section{B. Multiparticle collision dynamics}

The ambient fluid is described by the multiparticle collision (MPC) dynamics approach, an off-lattice, mesoscale, hydrodynamic simulation technique. ${ }^{54-56}$ In this method, the fluid is represented by point particles with positions $\boldsymbol{r}_{i}$ and velocities $\boldsymbol{v}_{\boldsymbol{i}}\left(i=1, \ldots, N_{s}\right)$. The particle dynamics proceeds in discrete steps, the streaming and collision step. During streaming, the fluid particles of mass $m$ move ballistically in a closed system. However, the gravitational-field induced backflow has to be taken into account, which yields the velocities and positions after streaming

$$
\begin{gathered}
\boldsymbol{v}_{i}(t+h)=\boldsymbol{v}_{i}(t)-\frac{M N_{p}}{M N_{p}+m N_{s}} \hat{\boldsymbol{G}} h, \\
\boldsymbol{r}_{i}(t+h)=\boldsymbol{r}_{i}(t)+h \boldsymbol{v}_{i}(t)-\frac{M N_{p}}{M N_{p}+m N_{s}} \hat{\boldsymbol{G}} \frac{h^{2}}{2},
\end{gathered}
$$

with the collision time $h$. In the collision step, the simulation box is partitioned into cubic cells of side length $a$ to define the multiparticle collision environment. The solvent particles are sorted into these cells and their relative velocities, with respect to the center-of-mass velocity of the cell, are rotated around a randomly oriented axis by an angle $\alpha$, i.e.,

$$
\boldsymbol{v}_{i}(t+h)=\boldsymbol{v}_{i}(t)+(\mathbf{R}(\alpha)-\mathbf{I})\left(\boldsymbol{v}_{i}(t)-\boldsymbol{v}_{c m}(t)\right),
$$

where $\mathbf{R}$ is the rotation matrix, $\mathbf{I}$ is the unit matrix, and $\boldsymbol{v}_{c m}=\sum_{j=1}^{N_{c}} \boldsymbol{v}_{j} / N_{c}$ is the center-of-mass velocity of the cell with $N_{c}$ particles. In this stochastic process, mass, momentum, and energy are conserved. Momentum conservation ensures hydrodynamic behavior which emerges on larger length and time scales. ${ }^{55,56,78}$

The interaction of the star polymers with the fluid is established during the collision step. ${ }^{56,57,65,79}$ Thereby, the bead velocities are rotated according to Eq. (7) similar to those of the fluid particles, with the center-of-mass velocity of the respective collision cell

$$
\boldsymbol{v}_{c m}(t)=\frac{\sum_{i=1}^{N_{c}} m \boldsymbol{v}_{i}(t)+\sum_{k=1}^{N_{c}^{m}} M \boldsymbol{V}_{k}(t)}{m N_{c}+M N_{c}^{m}} .
$$

Here, $N_{c}^{m}$ is the number of beads in the considered cell. Thereby, momentum is redistributed between the fluid and monomers, and long-range correlations emerge. ${ }^{59}$

In order to maintain a constant temperature and to remove the energy introduced by the external field, we apply the 
Maxwell-Boltzmann scaling (MBS) method, which yields a Maxwell-Boltzmann distribution of the fluid-particle velocities. ${ }^{80,81}$ In the MBS thermostat, the relative velocities-with respect to the center-of-mass velocity of a collision cell-of all particles within such a cell are scaled by a stochastic factor, leaving the dynamical properties of the system unaltered. The stochastic factor is determined from the Gamma distribution function of the kinetic energy of the particles in a cell.

\section{Parameters}

The dynamical behavior of the fluid depends on the various model parameters. The transport properties of the solvent are determined by the collision time $h$, the rotation angle $\alpha$, and the average number of particles $\left\langle N_{c}\right\rangle$ per cell, $55,56,82-86$ which corresponds to the fluid mass density $\rho_{s}=m\left\langle N_{c}\right\rangle / a^{3}$. Small collision times and a large number of MPC particles, $\left\langle N_{c}\right\rangle$, result in fluid-like behavior with a high Schmidt number $S c$. In our simulation, we choose parameters such that the transport of momentum due to collision dominates over diffusion. Explicitly, we use the collision time $h / \sqrt{m a^{2} /\left(k_{B} T\right)}=0.1$, the rotation angle $\alpha=130^{\circ}$, and $\left\langle N_{c}\right\rangle=10$. These parameters correspond to the solvent viscosity $\eta_{s}=8.7 \sqrt{m k_{B} T / a^{4}}$, kinematic viscosity $v_{s}=\eta_{s} / \rho_{s}=0.87 \sqrt{a^{2} k_{B} T / m}$, and the Schmidt number $S c \approx 17 .^{81}$

We study the sedimentation behavior of star polymers with the polymer arm lengths $N_{m}=10,20,40$, and 80. In order to achieve a comparable finite-size effect for the various polymer lengths on the dynamical quantities, we fix the ratio of the simulation box size along the field direction ( $y$-axis) and the radius of gyration of the star polymer for the respective arm length. A polymer gets elongated in the field direction, thus the size of the simulation box along the field direction has to be larger than the polymer length. Explicitly, we apply the following extensions $\left(L_{x}, L_{y}, L_{z}\right)$ of the simulation box for the various polymer lengths: $N_{m}=80, L_{x} / a=80, L_{y} / a=200$, $L_{z} / a=80 ; N_{m}=40, L_{x} / a=60, L_{y} / a=130, L_{z} / a=60 ; N_{m}$ $=20$; and $N_{m}=10, L_{x} / a=40, L_{y} / a=90, L_{z} / a=40$. Periodic boundary conditions are applied in all spatial directions. This corresponds to nearly $10^{7}$ fluid particles for the polymer length $N_{m}=80$ and nearly $10^{5}$ fluid particles for $N_{m}=10$. In general, $m N \gg M N_{s}$, hence the correction term for backflow [Eq. (4)] is typically negligible. All the simulations are performed over a range of field strength $G=M \hat{G} l / k_{B} T$, where $10^{-4} \leq G<1$.

For the polymer, we use the Lennard-Jones parameters $\epsilon$ $=k_{B} T$ and $\sigma / l=0.8$. The parameters for the harmonic bonds are $l=a$ and $k_{s} /\left(k_{B} T / l^{2}\right)=5000$. The mass of a bead is $M$ $=10 \mathrm{~m}$. The size of the central bead and the bond lengths to the respective first bead of a polymer arm are twice as large as those of the polymers themselves. This is necessary to allow for a large number of arms to be connected to the central bead.

The velocity Verlet algorithm ${ }^{77}$ is used to integrate Newton's equations of motion of the star polymer with the integration time step $h / 20$.

For an efficient simulation of the system, we apply a hybrid procedure, where a graphics processing unit (GPU) is combined with a central processing unit (CPU). MPC is the most time-consuming part of our simulation. Hence, we divide the computational task into two parts. The equations of motion of the star polymer are always integrated on the CPU. The MPC dynamics is performed on a GPU. Since MPC streaming of the fluid particles as well as their collisional interactions are carried out independently, the fluid dynamics is highly parallelizable and can be managed in an efficient way on a GPU. After every MPC streaming step, velocities and positions of the monomers are transferred from the CPU to the GPU for the collisional interaction with the fluid particles. After the collision with fluid, velocities are transferred back to the CPU for the integration of the bead equations of the solute. A detailed description of the GPU implementation of MPC is provided in Ref. 87.

\section{RESULTS}

\section{A. Sedimentation coefficient}

Under the influence of the external field and in the stationary state, the star polymer drifts along the direction of the field, with a constant average velocity. Hence, the magnitude of the total external force $F_{T}=M N_{p} \hat{G}$ is equal to the magnitude of the frictional force, i.e.,

$$
M N_{p} \hat{G}=\gamma V_{c m},
$$

where $\gamma$ is the total friction coefficient of the star polymer and $\boldsymbol{V}_{c m}=\sum_{i=1}^{N_{p}}\left\langle\boldsymbol{V}_{i}\right\rangle / N_{s}$ is its center-of-mass velocity. The ratio of the center-of-mass velocity and the external force defines the sedimentation coefficient $S$, thus

$$
S \equiv \frac{V_{c m}}{\hat{G}}=\frac{M N_{p}}{\gamma} .
$$

Using Stokes relation, the friction coefficient $\left(\gamma=6 \pi \eta R_{h}\right)$ is proportional to the hydrodynamic radius $R_{h}$ of the star polymer. With the approximation of the hydrodynamic radius by the radius of gyration $R_{g}$, for which scaling arguments yield the relation

$$
R_{g} \sim l N_{m}^{v} f^{(1-v) / 2}
$$

with the critical exponent $v \approx 0.6,{ }^{46,88,89}$ the sedimentation coefficient should exhibit the scaling relation

$$
S \sim N_{m}^{1-v} f^{(1+v) / 2}
$$

at least for unperturbed star polymers at low external forces. Our simulation studies of Ref. 90 on the diffusive dynamics of star polymers of various functionalities confirm approximately the dependence $R_{g} \sim f^{(1-v) / 2}$, with $v \approx 0.63$ for the considered short polymers, but show a somewhat stronger dependence of the hydrodynamic radius on $f$, namely, $R_{h} \sim f^{\hat{\delta}}$ with $\hat{\delta}=0.29$ instead of 0.2 . We like to mention that in the free draining limit, the friction coefficient is proportional to $N_{p}$ and the sedimentation coefficient is independent of the star molecular weight.

Figure 1 displays sedimentation coefficients for various functionalities as a function of the scaled strength $G=M \hat{G} l / k_{B} T$ of the external field. Note that $l$ corresponds to the Kuhn length of the polymer. The curves are normalized by the respective asymptotic sedimentation coefficient $S_{0}$ in the limit of vanishing field. As expected, the sedimentation coefficient is independent of $\hat{G}$ in the linear response regime for all functionalities. To achieve accurate results, we have generated nearly 


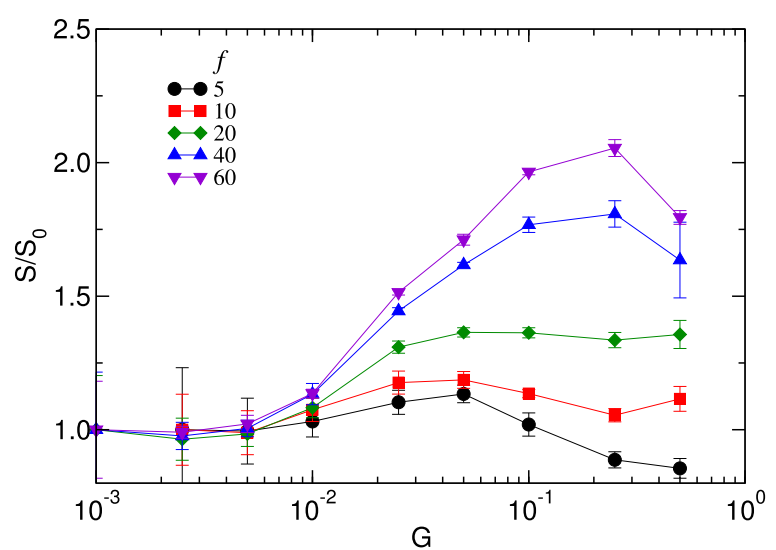

FIG. 1. Normalized sedimentation coefficients $S / S_{0}$ of star polymers with the indicated functionalities for the arm length $N_{m}=80$ as a function of the external field $G=M \hat{G} l /\left(k_{B} T\right) . S_{0}$ is the sedimentation coefficient of the asymptotic weak-field limit.

50 independent data sets for every $f$ in the weak-field limit because here thermal fluctuations are strong and the drift is weak. In an intermediate regime, $S / S_{0}$ increases with increasing $G$, passes through a maximum, and decreases again. Since we are limited in the range of applicable forces, we cannot extent our studies to large $G$ and, hence, cannot comment on the behavior for asymptotically large values. However, we observe a strong dependence on the functionality. Thereby, $S / S_{0}$ increases with increasing $f$ for intermediate field strengths and the maximum shifts to larger $G$. This is certainly related to considerable conformational changes of the star polymer as illustrated in Fig. 2.

The dependence of the sedimentation coefficient $S_{0}$ on the arm length and number is shown in Fig. 3. The values of $S_{0}$ for various $N_{m}$ and $f$ are obtained in the linear response regime in the weak-field limit. In this regime, $S_{0}$ is independent of $G$. In accord with the scaling prediction of Eq. (12), $S_{0}$ increases with increasing $N_{m}$ and $f$, respectively. Thereby, we find $S_{0} \sim N_{m}^{0.37}$ in close agreement with Eq. (12) for $v \approx 0.63$. The latter value is

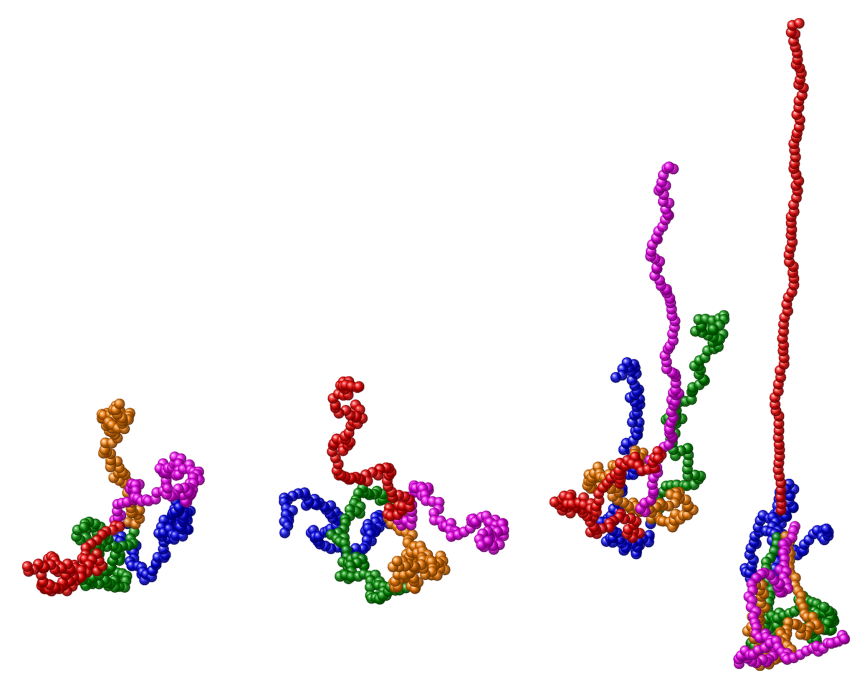

FIG. 2. Snapshots of a sedimenting star polymer for the arm number $f=5$, arm length $N_{m}=80$, and several values of the scaled gravitational field strength $G=10^{-3}, 10^{-2}, 10^{-1}$, and 0.5 (left to right). See also movies in the supplementary material.
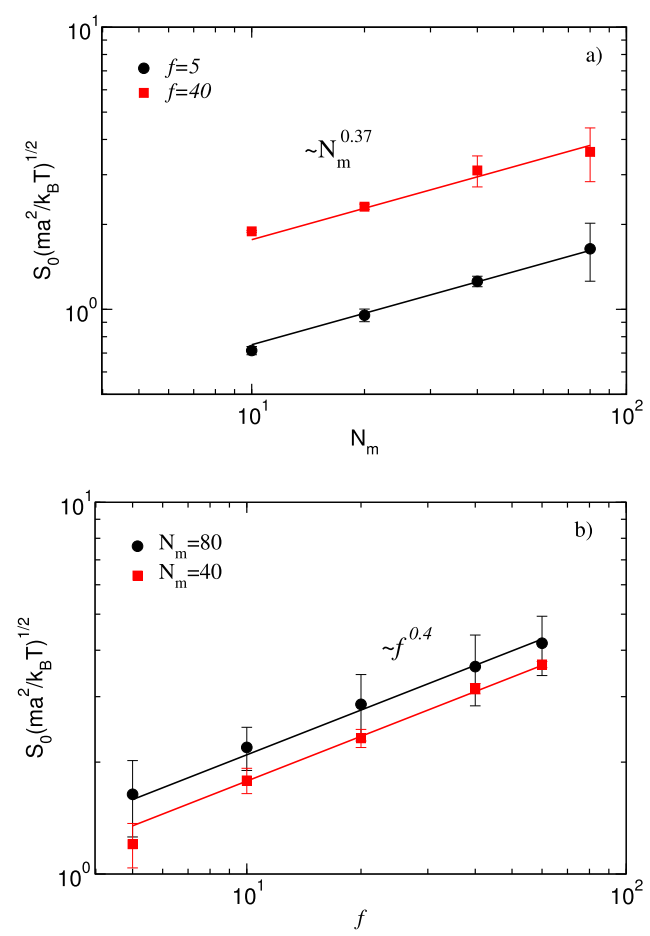

FIG. 3. (a) Dependence of the sedimentation coefficient $S_{0}$ on the length of polymer arms for $f=5$ and $f=40$. The solid lines illustrate the power-law dependence $S_{0} \sim N_{m}^{1-v}$, where $v \approx 0.63$. (b) Dependence of the sedimentation coefficient $S_{0}$ on the number of polymer arms $f$ for the arm lengths $N_{m}=40$ and $N_{m}=80$. The solid lines are the power-law fits $S_{0} \sim f^{\delta}$, with $\delta \approx 0.4$.

consistent with various simulation studies of equilibrium and nonequilibrium properties of star polymers for the considered arm lengths. ${ }^{49,51,67,90}$ For the dependence of $S_{0}$ in $f$, we find the power law $S_{0} \sim f^{\delta}$, with $\delta \approx 0.4$ independent of polymer length. However, $\delta$ is significantly smaller than the value predicted by scaling considerations [Eq. (12)], which is 0.82 . Even if we consider the somewhat stronger dependence $R_{h} \sim f^{0.29}$ on functionality, the value $\delta=0.4$ is significantly smaller than the theoretical prediction. The origin of the discrepancy remains to be resolved, but backflow might influence the hydrodynamic interactions between the beads.

Figure 4 displays the scaled sedimentation coefficient $S / S_{0}$ for the polymer lengths $N_{m}=10,20,40$, and 80 and the two functionalities $f=5$ and 40 as functions of the Weissenberg number Wi. The Weissenberg number is defined as follows. At weak external fields, a star polymer experiences a shear force on its surface during sedimentation, which gives rise to the shear rate $\dot{\gamma} \sim V_{c m} / R_{g}$. Within the blob model of a star polymer, ${ }^{46,47}$ this leads to the scaling relation for $\dot{\gamma}$ in terms of the arm length and functionality

$$
\dot{\gamma} \sim \hat{G} N_{m}^{1-2 v} f^{v} .
$$

The relaxation of a polymer arm is dominated by the relaxation of its largest blob ${ }^{47}$ and, hence, we define a Weissenberg number via $W i=\dot{\gamma} \tau_{B}$, with the blob relaxation time $\tau_{B}$. In the presence of hydrodynamic interactions, $\tau_{B} \sim R_{B}^{3}$, where $R_{B}$ is the blob radius. The latter scales as $R_{B} \sim R_{g} f^{-1 / 2} \sim N_{m}^{v} f^{-1 / 2}$ with arm length and functionality. ${ }^{47}$ Thus, we finally obtain the scaling relation for the Weissenberg number

$$
W i \sim \hat{G} N_{m}^{1+v} f^{v-3 / 2} .
$$



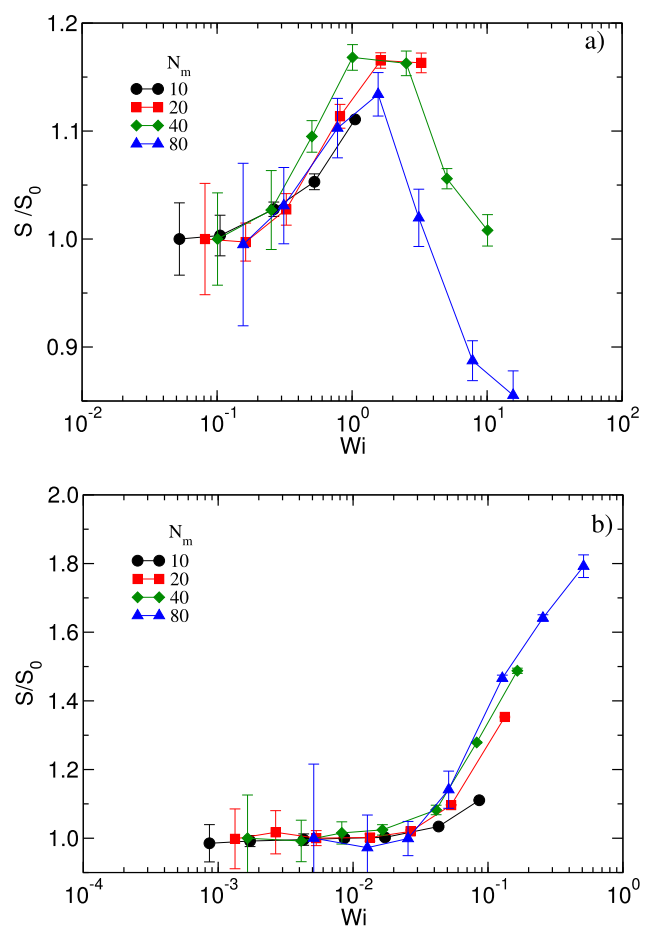

FIG. 4. Scaled sedimentation coefficients $S / S_{0}$ of star polymers with the indicated arm lengths as functions of the Weissenberg number Wi [Eq. (14)] for the arm numbers (a) $f=5$ and (b) 40 .

In the following, we present the sedimentation coefficient as a function of the Weissenberg number, taking $W i$ as $W i$ $=G N_{m}^{1+v} f^{v-3 / 2}$. As displayed in Fig. 4, a reasonable scaling of the curves for various arm lengths is only achieved for the functionality $f=40$ and longer arms. The predicted dependence on functionality is not reproduced by the simulations. This is not surprising, since the obtained scaling in Fig. 3(b) deviates from the simple scaling prediction.

As with increasing functionality, the ratio of $S / S_{0}$ increases with increasing external field strength in the nonlinear response regime. This increase is consistent with simulation results of linear polymers. ${ }^{44}$ The onset of the non-linear regime is reasonably well captured by prediction (14). As displayed in Fig. 4(b), in particular for the longer polymer arms, the sedimentation coefficient shows a non-monotonic dependence on the external field; it passes through a maximum value and then decreases again with increasing Wi. We expect a similar behavior for the shorter polymers; however, for them, we cannot reach large field strengths without violating limitations of the MPC method, e.g., small Mach numbers. The influence of the external field seems to be more pronounced for star polymers with a larger number of arms. Over the accessible range of field strengths, the values $S / S_{0}$ for star polymers of functionality $f=40$ are always higher than their linear-response-regime values [Fig. 4(b)], whereas the values of $S / S_{0}$ for $f=5$ are below the linear-response-regime values [Fig. 4(a)].

Considering the sedimentation velocities and the sizes of the star polymers, a remark on the Reynolds number is in order. Taking characteristic values for the sedimentation velocity and the radius of gyration, the Reynolds number $R e$ is $R e=2 S_{0} \hat{G} R_{g 0} / v \approx 10 G$ for $S_{0}=3$ and $R_{g 0}=15 a$. Hence, the Reynolds number is larger than unity for $G \gtrsim 0.1$. This implies that the observed saturation or weak decrease of the sedimentation coefficient (Fig. 1) appears for Reynolds numbers larger than unity. A priori, the effect of the Reynolds number on $S$ in this regime is not evident. A comparison of our results with those of Refs. 41 and 44 at zero Reynolds number for linear and ring polymers shows qualitative agreement $-S$ increases first with increasing $G$ and decreases again at larger $G$. Thereby, in Refs. 41 and 44 larger $G$ values are considered. Hence, from a qualitative point of view, we consider our results for star polymers as representative and expect a similar behavior for smaller Reynolds numbers. This is supported by Fig. 5, showing a non-turbulent flow field of a sedimenting star even at $R e$ $>1$. In any case, the simulation results for the larger $G$ values reflect the sedimentation behavior of star polymers at the respective Reynolds numbers.

\section{B. Structural properties}

Strong external fields induce large-scale conformational changes of the ultra-soft colloids, as illustrated in Fig. 2 for our star polymers. In order to characterize these conformational changes, we compute the radius-of-gyration tensor, which is defined as

$$
G_{\alpha \beta}=\frac{1}{N_{p}}\left\langle\sum_{i=1}^{N_{p}} \Delta R_{i \alpha} \Delta R_{j \beta}\right\rangle .
$$

Here, $\Delta R_{i \alpha}$ is the position of the $i$ th bead relative to the star center-of-mass and $\alpha, \beta \in\{x, y, z\}$. In the limit of a vanishing field, a star polymer is isotropic and all the diagonal

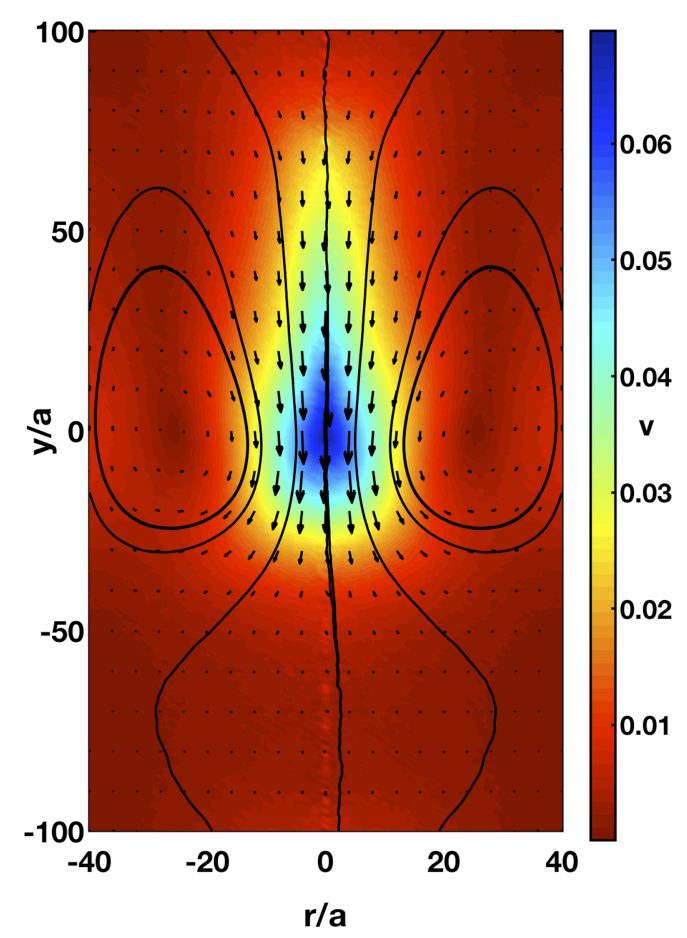

FIG. 5. Flow field of a star polymer in the laboratory reference frame for the arm length and number $N_{m}=80, f=60$, and field strength $G=0.5$. The coordinate $r$ is the radial distance with respect to the sedimentation direction ( $y$ axis). The star polymer drags along the fluid, which is indicated by the velocity-field vectors and solid black line. The flow lines in the head region reflect the recirculation of the fluid. ${ }^{41}$ Despite the strong field corresponding to Reynolds numbers larger than unity, the flow is laminar. 
components are equal, i.e., $G_{\alpha \alpha}=G_{\alpha \alpha}^{00}=R_{g 0}^{2} / 3$, where $R_{g 0}$ is the equilibrium radius of gyration [Eq. (11)].

Figure 6 shows normalized mean square radii of gyration $R_{g}^{2}$ and its components $R_{g L}^{2}$ and $R_{g T}^{2}$ along and transverse to the external field, respectively, as functions of the field strength $G$ for various functionalities. In the linear response regime, $G$ $\lesssim 2 \times 10^{-4}$, the size of a colloid is equal to its unperturbed equilibrium value. In an intermediate regime, $R_{g}^{2}$ decreases with increasing field strength. This compression of the ultrasoft colloid is more pronounced for high-functionality star polymers. Both, the transverse and longitudinal components $R_{g L}^{2}$ and $R_{g T}^{2}$ are reduced. In the case of $f \leq 10$, mainly $R_{g T}^{2}$ decreases with increasing $G$. We attribute this reduction in colloid size to hydrodynamics. The front of the star polymer experiences a drag force, which causes a compression. In addition, the flow surrounding the non-draining colloid exerts an inward force, which strongly affects $R_{g T}$. This is similar to the flow field of a linear polymer as discussed in Ref. 41.
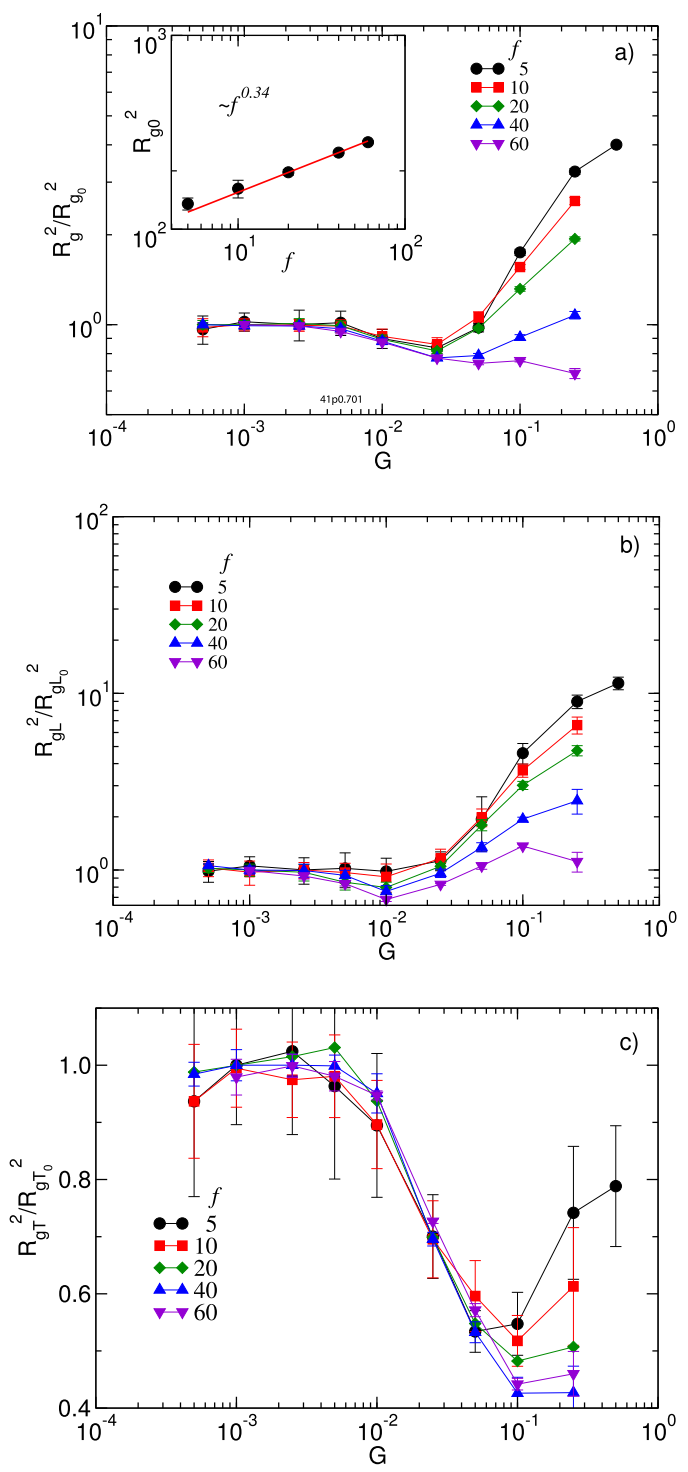

FIG. 6. (a) Overall radii of gyration of star polymers and their components (b) along and (c) perpendicular to the field as a function of the external field for the indicated functionalities and the arm length $N_{m}=80$. The inset in (a) shows the dependence of the equilibrium radius of gyration $R_{g 0}^{2}$ on the functionality $f$.
Above a functionality-dependent-field strength, the longitudinal component of the radius of gyration along with $R_{g}$ increases with increasing $G$. This increase is most pronounced for lowfunctionality star polymers. As illustrated in Fig. 2, in the limit of high fields, polymer arms are stretched, lag behind the center of the star polymer, and yield an increase of its radius of gyration. In this regime, the front-back symmetry of the star polymer is broken. Polymer arms in front of the star-polymer center are compressed, whereas arms behind the center are stretched significantly. This implies that the monomer density in the front core is higher and therefore also the gravitational pull. A similar anisotropic shape appears for other soft colloidal objects in a gravitational field, such as red blood cells. ${ }^{37}$ Within the accuracy of our simulations, the components of the radius-of-gyration tensor seem to approach constant values at large field strengths. [Note the pronounced fluctuations (error bars) of $R_{g T}^{2}$ at larger $G$ values due to large-scale shape changes of the head (cf. Fig. 8).] We attribute this, on the one hand, to the maximal possible stretching of the polymer arms and, on the other hand, a saturation of the compression of the major part of the star polymer by the fluid flow. The latter is to be expected for the transverse component of the radius of gyration, since excluded-volume interactions allow for a minimal size only.

The inset in Fig. 6(a) shows the dependence of the equilibrium radius of gyration on the functionality for the arm length $N_{m}=80$. The solid line indicates the power-law dependence $R_{g}^{2} \sim f^{1-v}$, with the exponent $v \sim 0.63$, which is consistent with the theoretical expectation according to Eq. (11).

The dependence of the star-polymer radius of gyration on the polymer arm length is displayed in Fig. 7 for $f=5$ and $f$ $=40$ as a function of the Weissenberg number. Here, we find good agreement between the curves for the various arm lengths as long as flow leads to a shrinkage of the star polymers. The appearance of strongly extended tails breaks the universality. Again, the predicted scaling relation (14) fails to describe the obtained functionality dependence. For short arm lengths, we observe a monotonic decrease of the star-polymer size and a crossover to a non-monotonic behavior for longer arms. In the case of short polymers $\left(N_{m} \lesssim 20\right)$, the radius of gyration and its components $R_{g L}$ and $R_{g T}$ always decreases for all Wi and both functionalities over the considered range of external field strengths. The size of the longer-arm star polymers increases again at higher field strengths due to the appearance of strongly stretched polymers. We expect such an increase for all polymer lengths. There is a critical field strength, which has to be exceeded to achieve the increase in size. This critical field strength depends on the arm length and the functionality and seems to be different for the longitudinal and transverse parts of the radius of gyration. Whereas $R_{g L}^{2}$ clearly increases for $N_{m}$ $\gtrsim 40$ and $W i>1(f=5)\left[W i>10^{-1}(f=40)\right]$, the respective values for the other arm lengths still decrease. The transverse components of the radii of gyration behave rather similarly, and $R_{g T}^{2}$ increases again for $W i \gtrsim 1$. Thereby, the relative change in $R_{g}^{2}$ for the fewer-arm stars is always larger than that of the higher functionality stars.

The star-polymer structure is highly dynamic. Although the overall shape is rather stable for a long time with the 

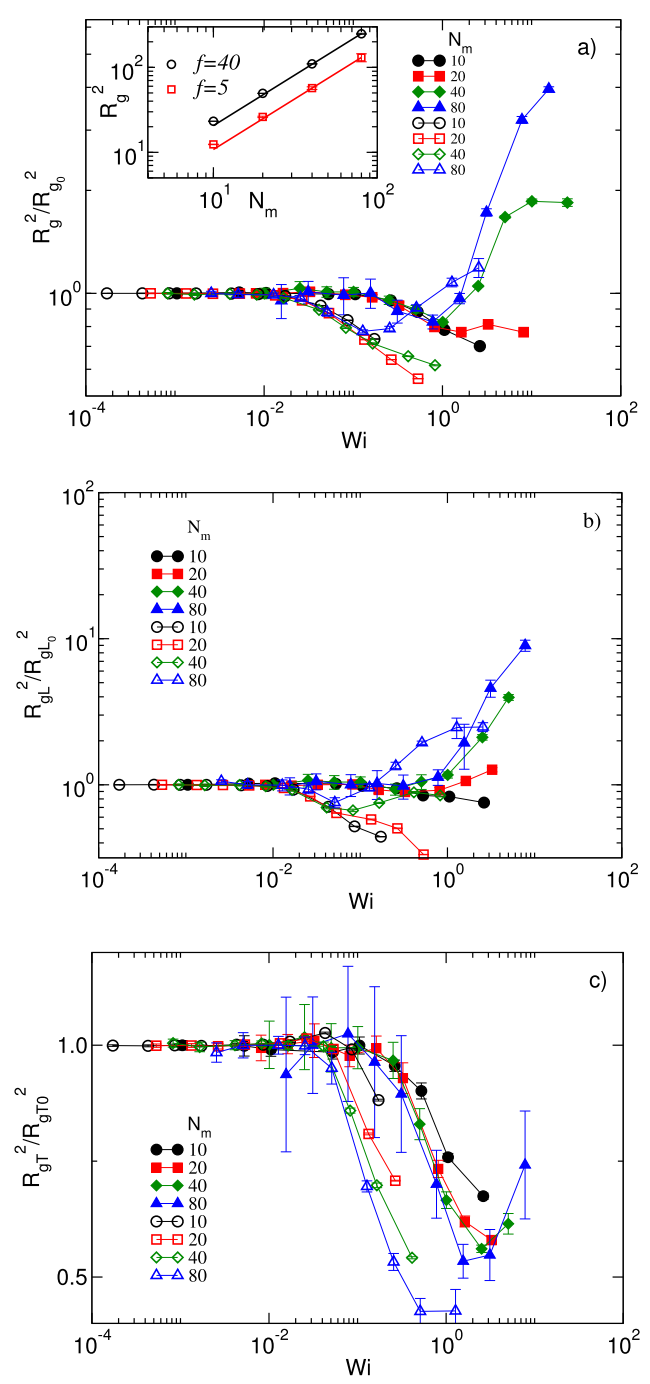

FIG. 7. (a) Overall radii of gyration of star polymers and their components (b) along and (c) perpendicular to the field as a function of $W i$ for the indicated arm lengths. Open symbols correspond to $f=40$ and closed symbols to $f=5$. The inset in (a) shows the dependence of the equilibrium radius of gyration $R_{g 0}^{2} \sim N_{m}^{2 v}$ on the arm length $N_{m}$, where $v$ $=0.63$.

majority of polymer arms close to the center of mass and an extended trailing tail, ${ }^{41,44}$ the individual polymers undergo considerable conformational changes. This is illustrated in Fig. 8 . For a movie, see the supplementary material. The emergence of an extended tail leads to an increase of the radii of gyration $R_{g}^{2}$ and $R_{g L}^{2}$. This increase is more pronounced for low-functionality star polymers as reflected in Figs. 6 and 7. For high-functionality star polymers, the relative weight of the small number of arms $(\lesssim 5)$ in the trailing tail is less important than for low-functionality star polymers. More remarkable is the increase of $R_{g T}$ at large field strengths. Here, the flow field seems to perturb the lower-field flow-induced compact structure and implies larger conformational changes of the polymers.

We have shown that the symmetry of the colloidal structure of the star polymer is broken in the limit of high field strengths. The asymmetric distribution of polymer arms in the high-field limit can be qualitatively illustrated by the probability distribution of the center-to-end distance of the
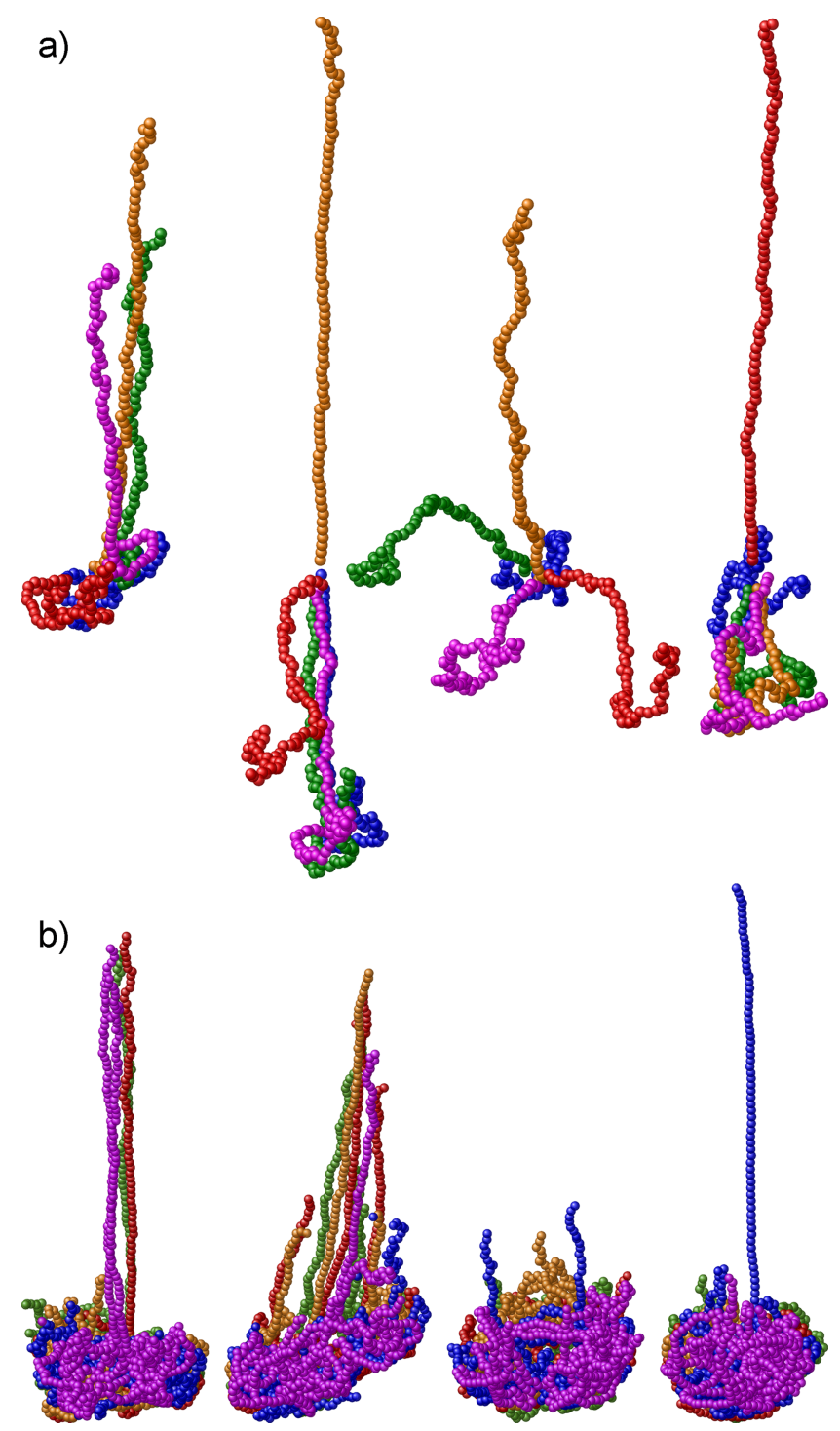

FIG. 8. Snapshots of sedimenting star polymers for different times. The arm length is $N_{m}=80$, the strength of the external field is $G=0.5$, and the arm numbers are $f=5$ (a) and $f=40$ (b). See also movies in the supplementary material.

polymer arms. As an example, Fig. 9 shows the normalized probability distribution function $P\left(R_{c e}\right)$ of the center-to-end distance of polymer arms for various field strengths. In the weak-field limit, the distribution of the center-to-end distance exhibits a peak at $R_{c e} / l \approx 20$ corresponding to the equilibrium value. With increasing $G$, the peaks shifts toward smaller values of the center-to-end distance and broaden substantially for large values of $G$ due to compressive force. At the same time, the probability for extended polymer arms increases. This signifies that on average a few arms are stretched while the majority of arms are compressed. In the limit of strong fields, the distribution function exhibits two peaks, at $R_{c e} / l \approx 10$ and $R_{c e} / l \approx 80$. The smaller peak at $R_{c e} / l \approx 80$ corresponds to nearly fully stretched arms. The height of the peak, smaller than the small-distance peak, reflects that only a few arms are strongly stretched but that these stretched conformations are rather stable and persistent. Due to fluctuations, stretched arms collapse and are replaced by others. Thereby, the collapse process is very fast, which leads to a low probability in the range $40 \lesssim R_{c e} / l<70$. 


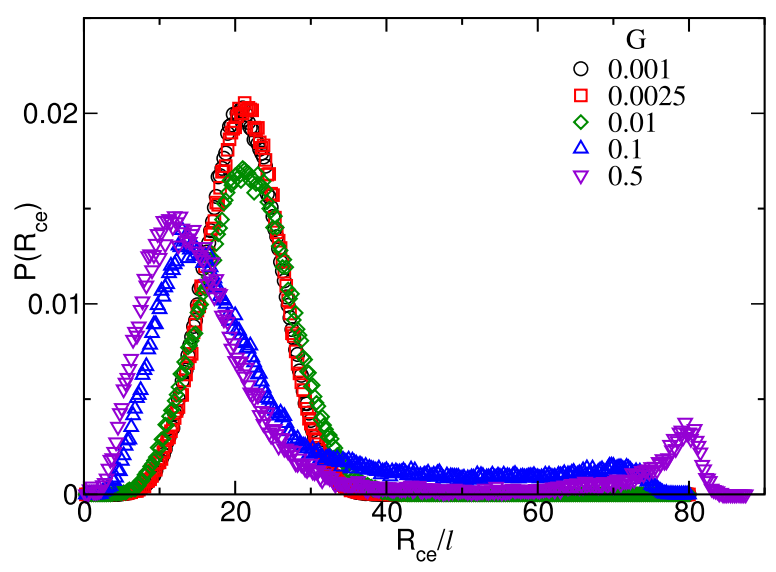

FIG. 9. Normalized probability distribution function $P\left(R_{c e}\right)$ of the centerto-end distance of polymer arms of length $N_{m}=80$ and the star-polymer functionality $f=40$ for various field strengths $G$ (as indicated).

\section{SUMMARY AND CONCLUSIONS}

We have investigated the steady-state sedimentation properties of ultra-soft colloids (star polymers) via hybrid mesoscale computer simulations. We find that the mobility of the ultra-soft colloid exhibits a non-monotonic dependence on the external field strength $G$. The sedimentation coefficient reaches a maximum value at an intermediate range of $G$. Thereby, for star polymers with fewer arms, the maximum appears at smaller field strengths and the sedimentation coefficient assumes smaller values in the high-field regime than the asymptotic value in the limit of vanishing field. The non-monotonic behavior of the sedimentation coefficient is related to flow-induced conformational changes of the star polymers. The increase of the sedimentation coefficient follows from a decrease of the radius of gyration. In the limit of high field strengths, the mobility decreases due to the stretching of various polymer arms along the field direction. Hence, the nonequilibrium dynamical properties of the star polymers are tightly linked with their structure. In the limit of strong external fields, the star polymers are no longer spherically symmetric. They rather exhibit a compact advancing structure, which is followed by a tail of a few polymer arms. The number of polymers in the tail strongly fluctuates and depends on the functionality.

The nonmonotonic behavior of the dynamical and structural properties appears in our simulations for $R e \gtrsim 1$. Nevertheless, we expect a very similar behavior for Reynolds numbers significantly smaller than unity. Our expectation is supported by the zero-Reynolds-number simulations of Refs. 41 and 44 , where linear and ring polymers exhibit a qualitatively similar behavior.

For weak fields, the star polymers sediment while maintaining their equilibrium shape, and the sedimentation coefficient exhibits the arm length and functionality dependence $S_{0}$ $\sim N_{m}^{1-v} f^{\delta}$, with $\delta \approx 0.4$. An additional speed-up with increasing arm number is obtained for stars in the non-linear regime over a certain range of gravitational constants, with trailing polymer arms. An additional factor is also obtained in the nonlinear regime for different arm lengths. However, the increase or even decrease of $S$ is less pronounced by varying the arm length than by varying the functionality. Hence, non-linear effects can enhance sedimentation and promote separation of star polymers of different sizes.

\section{SUPPLEMENTARY MATERIAL}

See supplementary material for two movie files which are provided to illustrate the star-polymer conformations. Movie S1: A sedimenting star polymer is shown with respect to its centre bead for the functionality $f=5$ and $G$ $=0.5$ at the arm length $N_{m}=80$. Movie S2: The same set of parameters are applied as in $S 1$, except the number of arms is $f=40$.

\section{ACKNOWLEDGMENTS}

Financial support by the Deutsche Forschungsgemeinschaft (DFG) through the Collaborative Research Center "Physics of Colloidal Dispersions in External Fields" (SFB TR6), by the EU through the Collaborative Research Project "NanoDirect" (No. NMP4-SL-2008-213948), and by the EU through FP7-Infrastructure ESMI (Grant No. 262348) is gratefully acknowledged. The authors gratefully acknowledge the computing time granted on the supercomputers at Jülich Supercomputing Centre (JSC).

\section{APPENDIX: FLUID BACKFLOW}

The equation of motion of a monomer of the star polymer in the laboratory reference frame (indicated by a prime) is given by

$$
M \ddot{\boldsymbol{R}}_{k}^{\prime \mu}=\boldsymbol{F}_{k}^{\mu}+M \hat{\boldsymbol{G}}
$$

where the $\boldsymbol{F}_{k}^{\mu}$ are intramolecular forces following from potentials (1) and (2). The center-of-mass velocity of the total system, star-polymer plus MPC fluid, is then

$$
M_{t o t} \ddot{\boldsymbol{r}}_{c m}^{\prime}=N_{p} M \hat{\boldsymbol{G}},
$$

with $M_{t o t}=N_{p} M+N_{s} m$ and

$$
\boldsymbol{r}_{c m}^{\prime}=\frac{1}{M_{t o t}}\left(\sum_{k, \mu} M \boldsymbol{R}_{k}^{\prime \mu}+\sum_{i} m \boldsymbol{r}_{i}^{\prime}\right) .
$$

Introducing the coordinates $\boldsymbol{R}_{k}^{\mu}=\boldsymbol{R}_{k}^{\prime \mu}-\boldsymbol{r}_{c m}^{\prime}$ and $\boldsymbol{r}_{i}=\boldsymbol{r}_{i}^{\prime}-\boldsymbol{r}_{c m}^{\prime}$ of the monomer and fluid particle positions with respect to the total center of mass implies

$$
\sum_{k, \mu} M \ddot{\boldsymbol{R}}_{k}^{\mu}+\sum_{i} m \ddot{\boldsymbol{r}}_{i}=0
$$

i.e., conservation of the total moment in the center-of-mass reference frame. We set this moment to zero initially. From Eq. (A1), we then obtain

$$
M \ddot{\boldsymbol{R}}_{k}^{\mu}=\boldsymbol{F}_{k}^{\mu}+M \hat{\boldsymbol{G}}+\boldsymbol{F}_{f},
$$

with

$$
\boldsymbol{F}_{f}=-\frac{M^{2} N_{p}}{M N_{p}+m N_{s}} \hat{\boldsymbol{G}}
$$

Similarly, we obtain for the fluid particles

$$
m \ddot{\boldsymbol{r}}_{i}=\frac{m}{M} \boldsymbol{F}_{f} .
$$


${ }^{1}$ R. G. Larson, The Structure and Rheology of Complex Fluids (Oxford University Press, Oxford, NY, 1999).

${ }^{2}$ R. B. Bird, R. C. Armstrong, and O. Hassager, Dynamics of Polymeric Liquids: Fluid Mechanics, 2nd ed. (Wiley, New York, 1987).

${ }^{3}$ D. E. Smith, H. P. Babcock, and S. Chu, Science 283, 1724 (1999).

${ }^{4}$ P. LeDuc, C. Haber, G. Boa, and D. Wirtz, Nature 399, 564 (1999).

${ }^{5}$ M. Kröger, Phys. Rep. 390, 453 (2004).

${ }^{6}$ C. M. Schroeder, R. E. Teixeira, E. S. G. Shaqfeh, and S. Chu, Phys. Rev. Lett. 95, 018301 (2005).

${ }^{7}$ S. Gerashchenko and V. Steinberg, Phys. Rev. Lett. 96, 038304 (2006).

${ }^{8}$ R. G. Winkler, Phys. Rev. Lett. 97, 128301 (2006).

${ }^{9}$ R. M. Jendrejack, D. C. Schwartz, J. J. de Pablo, and M. D. Graham, J. Chem. Phys. 120, 2513 (2004).

${ }^{10}$ R. Chelakkot, R. G. Winkler, and G. Gompper, Europhys. Lett. 91, 14001 (2010).

${ }^{11}$ R. Chelakkot, R. G. Winkler, and G. Gompper, Phys. Rev. Lett. 109, 178101 (2012).

${ }^{12}$ D. Steinhauser, S. Köster, and T. Pfohl, ACS Macro Lett. 1, 541 (2012).

${ }^{13}$ M. Harasim, B. Wunderlich, O. Peleg, M. Kröger, and A. R. Bausch, Phys. Rev. Lett. 110, 108302 (2013).

${ }^{14}$ T. Pakula, D. Vlassopoulos, G. Fytas, and J. Roovers, Macromolecules 31, 8931 (1998).

${ }^{15}$ D. Vlassopoulos, G. Fytas, T. Pakula, and J. Roovers, J. Phys.:Condens. Matter 13, R855 (2001).

${ }^{16}$ A. Nikoubashman and C. N. Likos, Macromolecules 43, 1610 (2010).

${ }^{17}$ D. Vlassopoulos and M. Cloitre, Curr. Opin. Colloid Interface Sci. 19, 561 (2014).

${ }^{18}$ R. G. Winkler, D. A. Fedosov, and G. Gompper, Curr. Opin. Colloid Interface Sci. 19, 594 (2014).

${ }^{19}$ S. R. Keller and R. Skalak, J. Fluid Mech. 120, 27 (1982).

${ }^{20}$ H. Noguchi and G. Gompper, Phys. Rev. Lett. 93, 258102 (2004).

${ }^{21}$ V. Kantsler and V. Steinberg, Phys. Rev. Lett. 96, 036001 (2006).

${ }^{22}$ C. Misbah, Phys. Rev. Lett. 96, 028104 (2006).

${ }^{23}$ V. Lebedev, K. Turitsyn, and S. Vergeles, Phys. Rev. Lett. 99, 218101 (2007).

${ }^{24}$ P. M. Vlahovska and R. S. Gracia, Phys. Rev. E 75, 016313 (2007).

${ }^{25}$ H. Zhao and E. S. Shaqfeh, J. Fluid Mech. 674, 578 (2011).

${ }^{26}$ D. Abreu, M. Levant, V. Steinberg, and U. Seifert, Adv. Colloid Interface Sci. 208, 129 (2014).

${ }^{27}$ A. Lamura and G. Gompper, Europhys. Lett. 102, 28004 (2013).

${ }^{28}$ M. Abkarian, M. Faivre, and A. Viallat, Phys. Rev. Lett. 98, 188302 (2007).

${ }^{29}$ H. Noguchi and G. Gompper, Proc. Natl. Acad. Sci. U. S. A. 102, 14159 (2005).

${ }^{30}$ B. Kaoui, G. Biros, and C. Misbah, Phys. Rev. Lett. 103, 188101 (2009).

${ }^{31}$ J. Dupire, M. Socol, and A. Viallat, Proc. Natl. Acad. Sci. U. S. A. 109, 20808 (2012).

${ }^{32}$ I. V. Pivkin and G. E. Karniadakis, Phys. Rev. Lett. 101, 118105 (2008).

${ }^{33}$ J. L. McWhirter, H. Noguchi, and G. Gompper, Proc. Natl. Acad. Sci. U. S. A. 106, 6039 (2009).

${ }^{34}$ G. Tomaiuolo, M. Simeone, V. Martinelli, B. Rotoli, and S. Guido, Soft Matter 5, 3736 (2009).

${ }^{35}$ D. A. Reasor, J. R. Clausen, and C. K. Aidun, Int. J. Numer. Methods Fluids 68, 767 (2012)

${ }^{36}$ D. A. Fedosov, M. Peltomäki, and G. Gompper, Soft Matter 10, 4258 (2014).

${ }^{37}$ M. Peltomaki and G. Gompper, Soft Matter 9, 8346 (2013).

${ }^{38}$ S. E. Harding, A. J. Rowe, and J. C. Horton, Analytical Ultracentrifugation in Biochemistry and Polymer Science; Royal Society of Chemistry (Cambridge, UK, 1993).

${ }^{39}$ T. M. Laue and W. F. Stafford, Annu. Rev. Biophys. Biomol. Struct. 28, 75 (1993).

${ }^{40}$ K. B. Abbitt and G. B. Nash, Am. J. Physiol. 285, H229 (2003).

${ }^{41}$ X. Schlagberger and R. R. Netz, Macromolecules 41, 1861 (2008).

${ }^{42}$ B. Zimm and V. Schumaker, Biophys. Chem. 5, 265 (1976).

${ }^{43}$ D. Ertaş and M. Kardar, Phys. Rev. E 48, 1228 (1993).

${ }^{44}$ X. Schlagberger and R. R. Netz, Phys. Rev. Lett. 98, 128301 (2007).

${ }^{45}$ J. Stellbrink, J. Allgaier, M. Monkenbusch, D. Richter, A. Lang, C. Likos, M. Watzlawek, H. Löwen, G. Ehlers, and P. Schleger, in Trends in Colloid and Interface Science XIV, Volume 115 of Progress in Colloid and Polymer Science, edited by V. Buckin (Springer Berlin Heidelberg, 2000), pp. 88-92.
${ }^{46}$ G. S. Grest, K. Kremer, and T. A. Witten, Macromolecules 20, 1376 (1987).

${ }^{47}$ G. S. Grest, K. Kremer, S. T. Milner, and T. A. Witten, Macromolecules 22, 1904 (1989).

${ }^{48}$ M. Ripoll, R. G. Winkler, and G. Gompper, Phys. Rev. Lett. 96, 188302 (2006).

${ }^{49}$ S. P. Singh, D. A. Fedosov, A. Chatterji, R. G. Winkler, and G. Gompper, J. Phys.: Condens. Matter 24, 464103 (2012).

${ }^{50}$ S. P. Singh, R. G. Winkler, and G. Gompper, Phys. Rev. Lett. 107, 158301 (2011).

${ }^{51}$ S. P. Singh, A. Chatterji, G. Gompper, and R. G. Winkler, Macromolecules 46, 8026 (2013).

${ }^{52}$ S. Gupta, S. Kundu, J. Stellbrink, L. Willner, J. Allgaier, and D. Richter, J. Phys.: Condens. Matter 24, 464102 (2012).

${ }^{53}$ J. Sablic, M. Praprotnik, and R. Delgado-Buscalioni, Soft Matter 13, 4971 (2017).

${ }^{54}$ A. Malevanets and R. Kapral, J. Chem. Phys. 110, 8605 (1999).

${ }^{55}$ R. Kapral, Adv. Chem. Phys. 140, 89 (2008).

${ }^{56}$ G. Gompper, T. Ihle, D. M. Kroll, and R. G. Winkler, Adv. Polym. Sci. 221, 1 (2009).

${ }^{57}$ A. Malevanets and J. M. Yeomans, Europhys. Lett. 52, 231 (2000).

${ }^{58}$ C.-C. Huang, G. Gompper, and R. G. Winkler, J. Phys.: Condens. Matter 24, 284131 (2012).

${ }^{59}$ C. C. Huang, G. Gompper, and R. G. Winkler, J. Chem. Phys. 138, 144902 (2013).

${ }^{60}$ N. Kikuchi, A. Gent, and J. M. Yeomans, Eur. Phys. J. E 9, 63 (2002).

${ }^{61}$ M. A. Webster and J. M. Yeomans, J. Chem. Phys. 122, 164903 (2005).

${ }^{62}$ J. F. Ryder and J. M. Yeomans, J. Chem. Phys. 125, 194906 (2006).

${ }^{63}$ S. Frank and R. G. Winkler, Europhys. Lett. 83, 38004 (2008).

${ }^{64}$ S. Frank and R. G. Winkler, J. Chem. Phys. 131, 234905 (2009).

${ }^{65}$ C.-C. Huang, R. G. Winkler, G. Sutmann, and G. Gompper, Macromolecules 43, 10107 (2010).

${ }_{66}^{66}$ J. T. Padding and A. A. Louis, Phys. Rev. Lett. 93, 220601 (2004).

${ }^{67}$ D. A. Fedosov, S. P. Singh, A. Chatterji, R. G. Winkler, and G. Gompper, Soft Matter 8, 4109 (2012).

${ }^{68}$ A. Nikoubashman and C. N. Likos, J. Chem. Phys. 133, 074901 (2010).

${ }^{69}$ Y.-G. Tao and R. Kapral, Soft Matter 6, 756 (2010).

${ }^{70}$ A. Zöttl and H. Stark, Phys. Rev. Lett. 108, 218104 (2012).

${ }^{71}$ J. Elgeti and G. Gompper, Proc. Natl. Acad. Sci. U. S. A. 110, 4470 (2013).

${ }^{72}$ S. Y. Reigh, R. G. Winkler, and G. Gompper, Soft Matter 8, 4363 (2012).

${ }^{73}$ M. Theers and R. G. Winkler, Soft Matter 10, 5894 (2014).

${ }^{74}$ M. Theers, E. Westphal, G. Gompper, and R. G. Winkler, Soft Matter 12, 7372 (2016).

${ }^{75}$ M. Yang, A. Wysocki, and M. Ripoll, Soft Matter 10, 6208 (2014).

${ }^{76}$ J. Hu, A. Wysocki, R. G. Winkler, and G. Gompper, Sci. Rep. 5, 9586 (2015).

${ }^{77}$ M. P. Allen and D. J. Tildesley, Computer Simulation of Liquids (Clarendon Press, Oxford, 1987).

${ }^{78}$ C.-C. Huang, G. Gompper, and R. G. Winkler, Phys. Rev. E 86, 056711 (2012).

${ }^{79}$ K. Mussawisade, M. Ripoll, R. G. Winkler, and G. Gompper, J. Chem. Phys. 123, 144905 (2005).

${ }^{80}$ C.-C. Huang, A. Chatterji, G. Sutmann, G. Gompper, and R. G. Winkler, J. Comput. Phys. 229, 168 (2010).

${ }^{81}$ C.-C. Huang, A. Varghese, G. Gompper, and R. G. Winkler, Phys. Rev. E 91, 013310 (2015).

${ }^{82}$ T. Ihle and D. M. Kroll, Phys. Rev. E 67, 066706 (2003).

${ }^{83}$ N. Kikuchi, C. M. Pooley, J. F. Ryder, and J. M. Yeomans, J. Chem. Phys. 119, 6388 (2003).

${ }^{84}$ C. M. Pooley and J. M. Yeomans, J. Phys. Chem. B 109, 6505 (2005).

${ }^{85}$ H. Noguchi and G. Gompper, Phys. Rev. E 78, 016706 (2008).

${ }^{86}$ R. G. Winkler and C.-C. Huang, J. Chem. Phys. 130, 074907 (2009).

${ }^{87}$ E. Westphal, S. P. Singh, C.-C. Huang, G. Gompper, and R. G. Winkler, Comput. Phys. Commun. 185, 495 (2014).

${ }^{88}$ M. Daoud and J. Cotton, J. Phys. 43, 531 (1982).

${ }^{89}$ T. Birshtein, E. Zhulina, and O. Borisov, Polymer 27, 1078 (1986).

${ }^{90}$ S. P. Singh, C.-C. Huang, E. Westphal, G. Gompper, and R. G. Winkler, J. Chem. Phys. 141, 084901 (2014). 